\title{
Spatially Constrained Anti-Aliasing Filter Using Slepian Eigenfunction Window on the Sphere
}

\author{
Usama Elahi $^{\star}$ Zubair Khalid ${ }^{\dagger}$, Member, IEEE, and Rodney A. Kennedy ${ }^{\star}$,Fellow, IEEE \\ * Research School of Engineering, The Australian National University, Canberra, ACT 2601, Australia \\ $\dagger$ School of Science and Engineering, Lahore University of Management Sciences, Lahore, Pakistan \\ Email: \{usama.elahi@anu.edu.au, zubair.khalid@lums.edu.pk,rodney.kennedy@anu.edu.au\}
}

\begin{abstract}
Spherical microphone arrays sample the sound field on the sphere in both space and time. The performance of spherical microphone arrays is typically limited by spatial aliasing which introduces side-lobes in the array beam pattern. In order to reduce the aliasing error, anti-aliasing filters, both ideal and spatially constrained, have been presented in the literature. In this paper, we propose a slepian eigenfunction window which spatially truncate the ideal anti-aliasing filter used in literature to design a spatially constrained anti-aliasing filter. We also provide an illustration to show that the aliasing on the beam pattern is reduced by the use of the proposed anti-aliaisng filter and compare the results with the spatially constrained filters obtained by applying rectangular and hamming windows. Robustness analysis such as measurement of the white noise gain and directivity index shows the superiority of the proposed filter over other spatially constrained anti-aliasing filters.

Index Terms-Microphone arrays, spatial aliaisng, spatial truncation, robustness, anti-aliasing filters, window functions.
\end{abstract}

\section{INTRODUCTION}

Signals are naturally defined on the sphere in many real world applications, particularly, in acoustics, spherical microphone arrays have been used for beamforming [1], [2], sound field anlysis [3] and sound field recordings [4], [5]. In array processing, spherical harmonics transform (SHT) [6] has been used in the analysis of array performance [2]. A significant factor that limits the performance of a spherical array at high frequencies is spatial aliasing [7] which generates side lobes in the array beam pattern. In literature, in order to position microphones on a sphere, sampling schemes have been devised that are aliasing free for band-limited functions [8], however, acoustics sound field produced by

This work was supported under the Australian Research Council's Discovery Projects funding scheme (Project no. DP150101011 and Project no. DP170101897). Zubair Khalid is supported by Pakistan HEC 2016-17 NRPU (Project no. 5925). plane waves are not band-limited on the sphere, giving rise to spatial aliasing at higher frequencies in practice.

A theoretical analysis of the performance of spherical microphone array is presented in [7] and the analysis reveals that spatial aliasing is one of the significant factors affecting the performance. In order to improve the performance of the microphone array, anti-aliasing filters are studied in [8], where spatial truncation is applied on ideal filter in order to get spatially constrained antialiasing filters. For spatial truncation on the sphere many window functions have been proposed in literature [9].

In this work, we propose to use suitably selected slepian eigenfunction window for spatial truncation in order to get spatially constrained anti-aliasing filter from ideal filter designed in [8]. The proposed window is obtained as a solution of slepian concentration problem on the sphere [10], [11]. We calculate the array directivity by applying spatially constrained anti-aliasing filters using the proposed eigenfunction window, rectangular window and hamming window of same width and compare the results with those obtained by applying ideal filter. We also perform robustness analysis, such as white noise gain (WNG) and directivity index (DI) [12]. Our analysis shows that for the reduction of side lobes produced by spatial aliasing error, anti-aliasing filter spatially truncated by the proposed eigenfunction window is a better choice than the rectangular and hamming windows.

The paper is organised as follows. The mathematical background is given in Section II. Sampling scheme used, aliasing function and spatial filtering are reviewed in detail in Section III. Spectral or Spatial closed form expressions for rectangular window and hamming window are also presented. Construction of the proposed eigenfunction window and use of window functions in producing spatially constrained anti-aliasing filters are discussed in detail in Section III. In Section IV, an array example is provided to see the effect of aliasing and 
benefit of anti-aliasing filter. Performance parameters such as WNG and DI are also calculated. Concluding remarks are made in Section V.

\section{Signals AND SYSTEMS ON THE SPHERE}

In this paper, we consider complex separable Hilbert space of finite energy functions on the unit sphere, $L^{2}\left(\mathbb{S}^{2}\right)$, which are equipped with the following inner product [6]

$$
\langle f, g\rangle \triangleq \int_{\mathbb{S}^{2}} f(\theta, \phi) \overline{g(\theta, \phi)} \sin \theta d \theta d \phi,
$$

where $\overline{(\cdot)}$ represents the complex conjugate operation and $\sin \theta d \theta d \phi$ is the differential area element on the sphere. A point on the unit sphere is parameterized by $(\sin \theta \cos \phi, \sin \theta \sin \phi, \cos \theta) \in \mathbb{S}^{2} \subset \mathbb{R}^{3}$ where $\theta \in[0, \pi]$ denotes the co-latitude and $\phi \in[0,2 \pi)$ represents the longitude. The inner product (1) induces a norm $\|f\| \triangleq\langle f, f\rangle^{1 / 2}$ and functions with finite induced norms are referred to as signals on the sphere [6].

Spherical harmonics, denoted by $Y_{\ell}^{m}(\theta, \phi)$ of all integer degrees $\ell \geq 0$ and integer orders $m \leq|\ell|$ form archetype complete orthonormal set of basis functions for $L^{2}\left(\mathbb{S}^{2}\right)$, and therefore, we can expand a function $f \in L^{2}\left(\mathbb{S}^{2}\right)$ as

$$
f(\theta, \phi)=\sum_{\ell=0}^{\infty} \sum_{m=-\ell}^{\ell}(f)_{\ell}^{m} Y_{\ell}^{m}(\theta, \phi),
$$

where $(f)_{\ell}^{m}$ denotes the spherical harmonic Fourier coefficient of degree $\ell$ and order $m$ and is given by

$$
(f)_{\ell}^{m} \triangleq\left\langle f, Y_{\ell}^{m}\right\rangle=\int_{\mathbb{S}^{2}} f(\theta, \phi) \overline{Y_{\ell}^{m}(\theta, \phi)} \sin \theta d \theta d \phi .
$$

The signal $f(\theta, \phi)$ is considered as band-limited at degree $L$ if $(f)_{\ell}^{m}=0$ for all $\ell \geq L$.

\section{Spatial Sampling, Aliasing And Filtering ON THE SPHERE}

\section{A. Sampling Scheme}

In acoustics, the sound field is sampled spatially by microphone arrays, particularly spherical arrays perform spatial sampling of functions (such as sound pressure) defined on the sphere. In order to avoid aliasing, spatial sampling requires limited band-limit. In literature, for spherical microphone arrays, sampling schemes have been devised which provide zero or negligible aliasing for band-limited functions for a given band-limit $L$ [8], [13]. We adopt equiangular sampling scheme proposed in [14] as it requires least number of samples for exact computation of SHT defined in (3) of a band-limited signal on the sphere. We use $\Omega_{L}$ to denote the set of equiangular sampling points taken on $L$ iso-latitude rings placed at the following co-latitude positions

$$
\theta_{j}=\frac{2 \pi j}{2 L-1}, \quad j=0,1,2, \ldots, L-1,
$$

with $2 L-1$ samples along longitude in each ring given by

$$
\phi_{p}=\frac{2 \pi p}{2 L-1}, \quad p=0,1,2, \ldots, 2 L-2 .
$$

We note that the SHT of the signal band-limited at $L$ can be evaluated exactly by taking samples of the signal over $\Omega_{L}$ [14]. The regular angular difference in the equiangular sampling scheme is useful for taking spatial samples by a rotating microphone.

\section{B. Aliasing Function and Spatial Filtering}

The performance of spherical microphone arrays at high frequencies is significantly affected by spatial aliasing. Although in spherical array processing, in order to arrange microphones on a sphere, we have aliasing free sampling schemes for band-limited functions on the sphere. The sound pressure, however is not a bandlimited function on sphere and hence spatial aliasing is expected due to the higher frequency harmonics [5]. A detailed analysis of the nature of aliasing error in spherical microphone arrays is given in [7]. We sample the function (such as sound pressure) by $M$ microphone arrays at positions $\Omega_{t}$ and its spherical coefficients are estimated by using (2) as

$$
\begin{aligned}
(\hat{f})_{\ell}^{m} & =\sum_{t=1}^{M} \alpha_{t} f\left(\theta_{t}, \phi_{t}\right) \overline{Y_{\ell}^{m}\left(\theta_{t}, \phi_{t}\right)} \\
& =\sum_{\ell^{\prime}=0}^{\infty} \sum_{m^{\prime}=-\ell^{\prime}}^{\ell^{\prime}}(f)_{\ell^{\prime}}^{m^{\prime}} \sum_{t=1}^{M} \alpha_{t} Y_{\ell^{\prime}}^{m^{\prime}}\left(\theta_{t}^{\prime}, \phi_{t}^{\prime}\right) \overline{Y_{\ell}^{m}\left(\theta_{t}, \phi_{t}\right)}
\end{aligned}
$$

where $\alpha_{t}$ are the weights which depend on the sampling scheme chosen and

$\sum_{t=1}^{M} \alpha_{t} Y_{\ell^{\prime}}^{m^{\prime}}\left(\theta_{t}^{\prime}, \phi_{t}^{\prime}\right) \overline{Y_{\ell}^{m}\left(\theta_{t}, \phi_{t}\right)}=\left\{\begin{array}{l}\delta_{\ell \ell^{\prime}} \delta_{m m^{\prime}} \ell, \ell^{\prime} \leq L, \\ \epsilon_{\ell, m, \ell^{\prime}, m^{\prime}} \ell \leq L<\ell^{\prime},\end{array}\right.$

where $\delta_{\ell \ell^{\prime}} \delta_{m m^{\prime}}$ represent the Kronecker delta function and $\epsilon_{\ell, m, \ell^{\prime}, m^{\prime}}$ is the aliasing error.

As sound fields are not band-limited, aliasing errors are experienced in practice. High frequency harmonics $\left(\ell^{\prime}>L+1\right)$ significantly contribute to the aliasing 
error, therefore, in order to reduce the energy content of the input signal at high frequencies, anti-aliasing filters based on spatial filtering techniques are used before sampling.

Define two functions $g$ and $h$ on the unit sphere, $\mathbb{S}^{2}$, representing the sound pressure and the filter function respectively. In order to implement the process of spatial filtering, we rotate the microphone around the sphere and measure its output at each rotated position. The spatial filtering can be obtained by using spherical correlation as [15]

$$
T(\alpha, \beta, \gamma)=\int_{\mathbb{S}^{2}} g(\Omega) \overline{\Lambda(\alpha, \beta, \gamma) h(\Omega)} \sin \theta d \theta d \phi,
$$

where $\Omega \in \mathbb{S}^{2}$ represents the position on the sphere and $\Lambda(\alpha, \beta, \gamma)$ is the rotation operator in $S O(3)$, where $\alpha$ and $\gamma$ represents rotation along $\mathrm{z}$-axis and $\beta$ rotation along the y-axis. The spherical correlation in (8) can be re-written as [15]

$$
T(\Lambda)=\sum_{\ell=0}^{\infty} \sum_{m=-\ell}^{\ell} \sum_{m^{\prime}=-\ell}^{\ell}(g)_{\ell}^{m} \overline{(h)_{\ell}^{m} D_{\ell^{\prime}}^{m m^{\prime}}}(\Lambda),
$$

where $D_{\ell}^{m m^{\prime}}(\Lambda)$ are the wigner-D functions [6], which are basis functions for the fourier transform on the rotation group $S O(3)$. Note that the filter function $h$ has azimuthal symmetry along the azimuth and is invariant under rotation along the z-axis $(\gamma=0)$, that is, $\Lambda(\alpha, \beta, \gamma)=\Lambda(\alpha, \beta)$. Due to the azimuthally symmetric filter, $T$ in (9) is mapped to $\mathbb{S}^{2}$ instead of $S O(3)$ and

$$
\begin{aligned}
T(\alpha, \beta) & =\sum_{\ell=0}^{\infty} \sum_{m=-\ell}^{\ell}(g)_{\ell}^{m} \overline{(h)_{\ell}^{0} D_{\ell}^{m 0}}(\alpha, \beta, 0), \\
& =\sum_{\ell=0}^{\infty} \sum_{m=-\ell}^{\ell}(g)_{\ell}^{m} \overline{(h)_{\ell}^{0}} \sqrt{\frac{4 \pi}{2 \ell+1}} Y_{\ell}^{m}(\alpha, \beta), \\
& =\sum_{\ell=0}^{\infty} \sum_{m=-\ell}^{\ell}(T)_{\ell}^{m} Y_{\ell}^{m}(\alpha, \beta),
\end{aligned}
$$

where

$$
(T)_{\ell}^{m}=(g)_{\ell}^{m} \overline{(h)_{\ell}^{0}} \sqrt{\frac{4 \pi}{2 \ell+1}} .
$$

It can be seen that aliasing error will diminish if such a filter $h$ can be designed which has low or zero values at high frequencies in the harmonic domain. Following (11), an ideal antialiasing filter can be designed as

$$
(h)_{\ell}^{0}= \begin{cases}\sqrt{\frac{2 \ell+1}{4 \pi}} & 0 \leq \ell \leq L, \\ 0 & \ell>L\end{cases}
$$

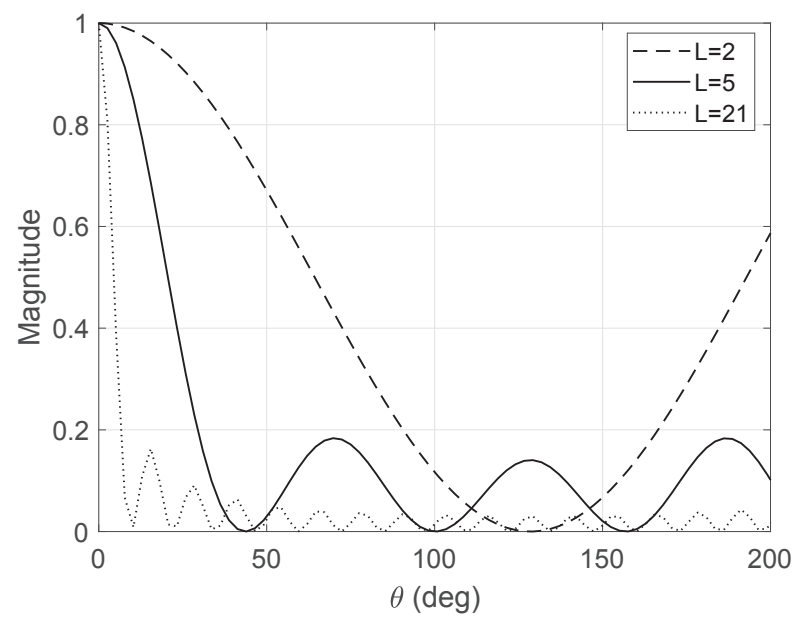

Fig. 1: Magnitude of the ideal anti-aliasing filter as a function of elevation angle $\theta$ for various values of $L$.

and the function $h$ can be obtained by taking inverse SHT as

$$
\begin{aligned}
h(\theta, \phi) & =\sum_{\ell=0}^{L} \sqrt{\frac{2 \ell+1}{4 \pi}} Y_{\ell}^{0}(\theta, \phi), \\
& =\frac{L}{4 \pi(\cos \theta-1)}\left[P_{L}(\cos \theta)-P_{L-1}(\cos \theta)\right] .
\end{aligned}
$$

Fig. 1 shows the magnitude of the ideal anti-aliasing filter as a function of the elevation angle $\theta$, for several values of $L$, normalized to have a unity gain at $\theta=0$.

\section{Spatially Constrained Anti-Aliasing Filters}

Using an ideal filter means building such a microphone which has a sensing surface covering the entire sphere which is not cost efficient. Practically, we want such a sensor which is more spatially constrained and cover only a small section of the sphere. In order to experience minimum possible aliasing, it is desirable to design such a spatially contrained filter whose performance matches an ideal filter. One way to design such a filter is to spatially truncate the ideal filter by applying a window of width $\theta_{o}$. In the following, we will study three type of windows namely Rectangular Window, Hamming window and the proposed slepian eigenfunction window [8], [9].

1) Rectangular Window : The rectangular window performs spectral truncation upto a certain band-limit, $\mathrm{L}$ and its spectral form is given by

$$
(h)_{\ell}^{0}= \begin{cases}1 & 0 \leq \ell \leq L, \\ 0 & \text { otherwise }\end{cases}
$$


2) Hamming Window: The hamming window in Eucledian domain [16] is defined in terms of the width of the main lobe $\theta_{o}$ as follows

$$
h\left(\theta, \theta_{o}\right)= \begin{cases}0.54+0.46 \cos \left(\pi \theta / \theta_{o}\right) & 0 \leq \theta \leq \theta_{o} \\ 0 & \text { otherwise }\end{cases}
$$

3) Slepian Eigenfunction Window: There exist such band-limited functions on the sphere that maximizes the propotion of energy that falls in the desired polar cap region, $\theta \in\left[0, \theta_{o}\right]$ and to find such functions one has to solve the slepian concentration problem on the sphere [9]-[11]. The eigenfunction window $h$ with band-limit $L$ and energy concentration within cap angle $\theta_{o}$ can be computed in spectral domain as a solution of following algebraic eigenvalue problem

$$
\mathbf{K h}=\lambda \mathbf{h},
$$

where $\mathbf{h}=\left[(h)_{0}^{0},(h)_{1}^{0}, \cdots,(h)_{\ell}^{0}\right]$ and $\mathbf{K}$ is real and symmetric matrix with dimensions $(L+1) \times(L+1)$ and its entries are given by

$$
K_{\ell, \ell^{\prime}}=2 \pi \int_{0}^{\theta_{o}} Y_{\ell}^{0}(\theta, 0) Y_{\ell^{\prime}}^{0}(\theta, 0) \sin \theta d \theta,
$$

which requires the computation of wigner- $3 j$ symbols and can be computed analytically. The eigenvalue problem in (16) can also be solved by eigen decomposition of a matrix $\mathbf{Q}$ of size $(L+1) \times(L+1)$ commuting it with $\mathbf{K}$, that is, $\mathbf{K Q}=\mathbf{Q K}$. $\mathbf{Q}$ is a tridiagonal matrix having the following entries [9]

$$
\begin{aligned}
Q_{\ell, \ell+1} & =\frac{(\ell+1)((\ell(\ell+2)-(L)(L+2))}{\sqrt{(2 \ell+1)(2 \ell+3)}}, \\
Q_{\ell, \ell} & =-\ell(\ell+1) \cos \left(\theta_{o}\right), \quad Q_{\ell, \ell^{\prime}}=0 .
\end{aligned}
$$

The eigen decomposition of $\mathbf{Q}$ gives $L+1$ eigen vectors of the form $\mathbf{h}$ and one with the largest eigenvalue gives the spectral domain representation, $(h)_{\ell}^{0}$ of desired eigenfunction window. Note that neither the spatial nor the spectral form are closed forms but can be obtained easily by eigen decomposition of $\mathbf{Q}$.

For $L=5$, it is observed from Fig. 1 that ideal filter designed in (13) has significant values below the first zero at $\theta=44^{\circ}$. In order to design spatially constrained filter, we apply rectangular, hamming and proposed eigenfunction windows of width $\theta_{o}=44^{\circ}$ to the ideal filter in (13). Fig. 2 shows the magnitude of the spherical harmonic coefficients of these spatially constrained windows plotted against degrees $\ell$ and for

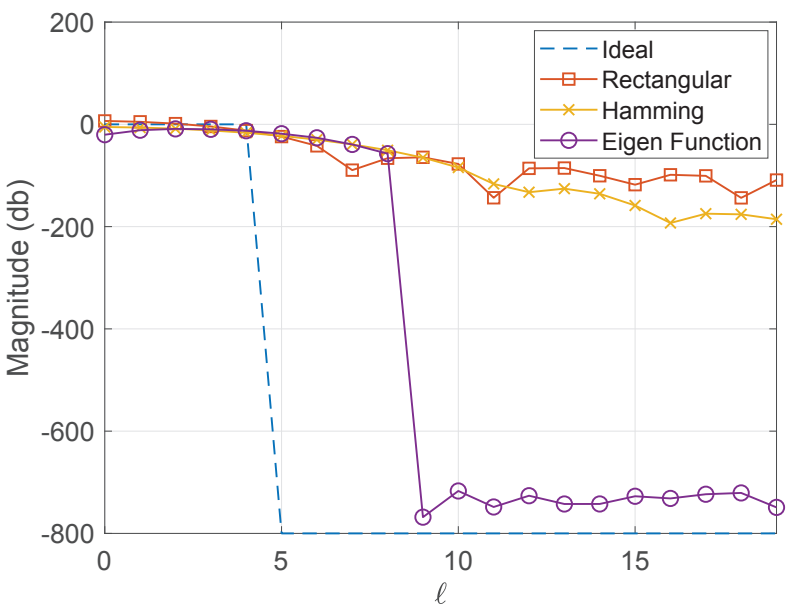

Fig. 2: Magnitude of ideal anti-aliasing filter for $L=5$ and spatially constrained filters using rectangular window, hamming window and proposed slepian eigenfunction window of width $\theta_{o}=44^{\circ}$.

comparison the coefficients of ideal filter are also plotted. It can be seen that eigenfunction windows out-performs rectangular and hamming windows attenuating most of the higher frequency harmonics above $\ell=L$. Note that increasing the window width will further improve the attenuation and reduce the overall aliasing error.

\section{AnAlysis}

\section{A. Array Processing}

In this example, we consider a spherical microphone array which samples the sound pressure, $p(k r, \Omega)$ of a plane wave sound field on the sphere, where $k$ is the wave number and $r$ is the radius of the rigid sphere [12]. A single unit amplitude plane wave is assumed to be arriving from direction $\Omega_{0}=(\pi / 2,0)$ and the plane wave decomposition is analysed in directions, $\Omega_{t} \in(\pi / 2,[0,2 \pi])$. In array processing, aliasing errors are produced at high frequencies, particularly for $k r>7$ [8]. In this example, significant aliasing errors are expected as a fourth order array with frequency $k r=7$ is analysed. The array output for a practical array of order $\ell \leq L$ is calculated as

$$
\begin{aligned}
y & =\int_{\mathbb{S}^{2}} p(k r, \Omega) \overline{w(\Omega)} \sin \theta d \theta d \phi, \\
& =\sum_{\ell=0}^{L-1} \sum_{m=-\ell}^{\ell}(p)_{\ell}^{m}(k r) \overline{w_{\ell}^{m}(k r)},
\end{aligned}
$$

where $(p)_{\ell}^{m}(k r)=b_{\ell}(k r) Y_{\ell}^{m}\left(\Omega_{o}\right)$ are the estimated spherical harmonic coefficients of sound pressure, $p$ and 


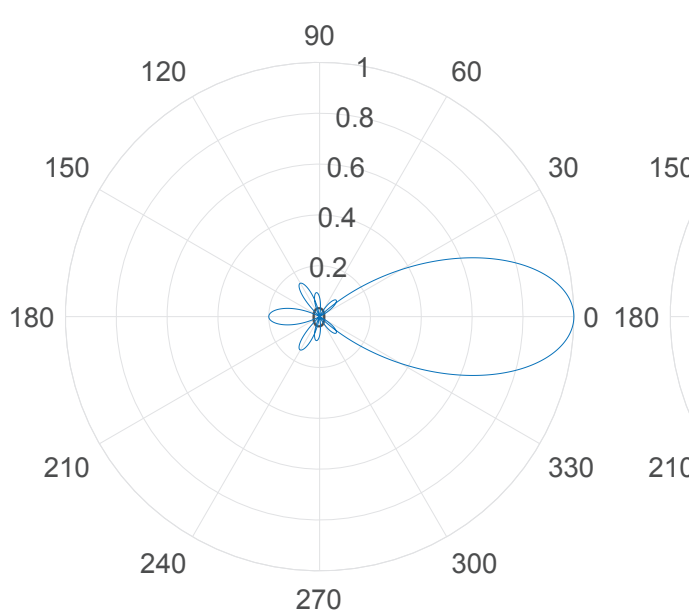

(a)

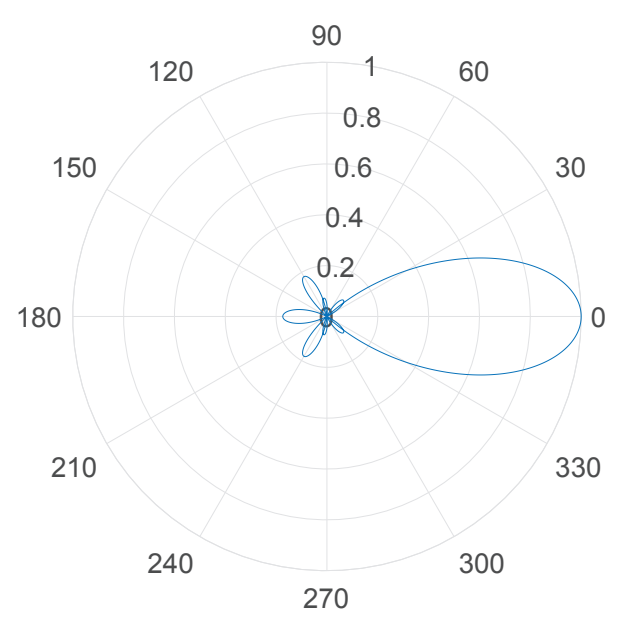

(d)

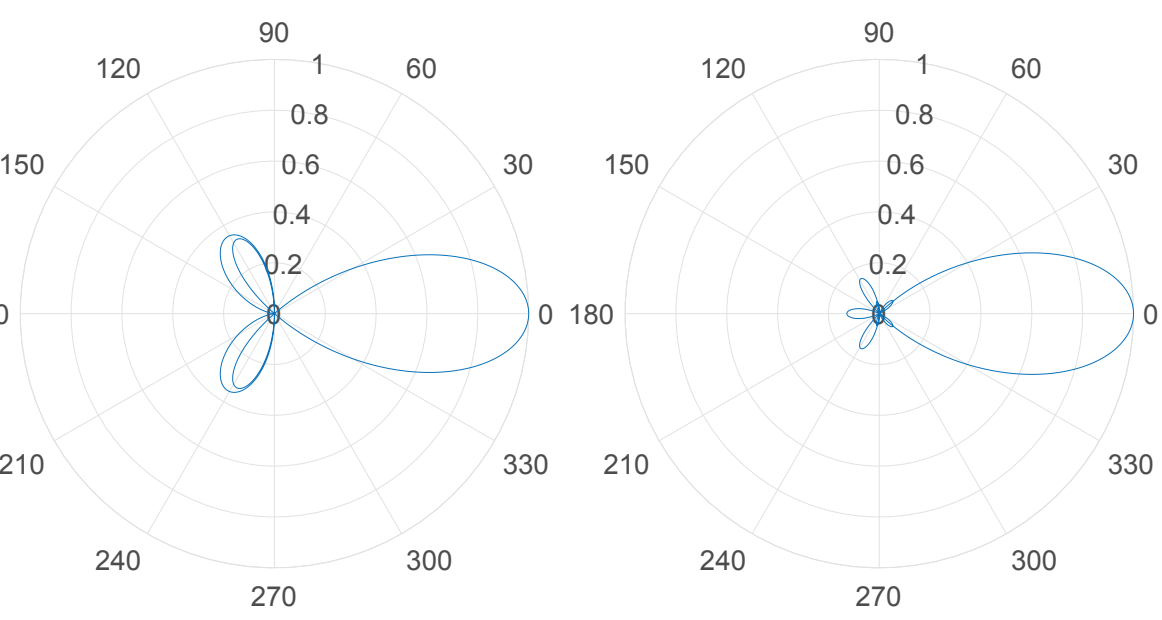

(b) (c)

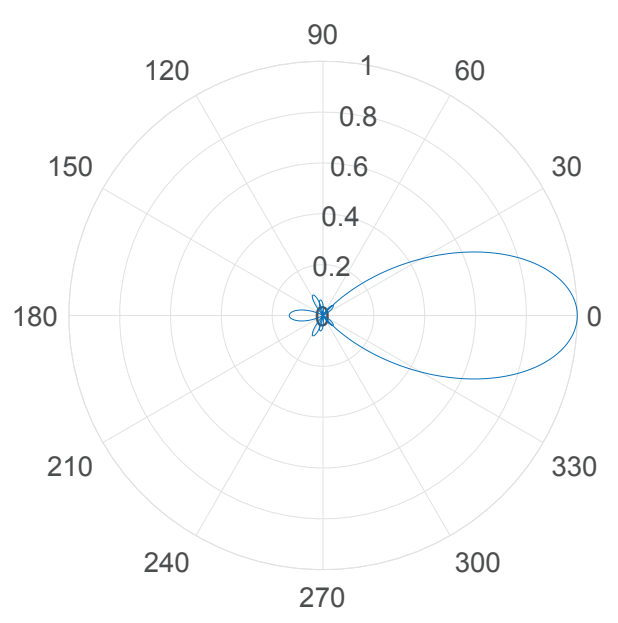

(e)

Fig. 3: Magnitude of the fourth order plane wave decomposition array directivity with (a) ideal sampling, no aliasing error (b) sampling without anti-aliasing filter (c) sampling with rectangular window spatially constrained anti-aliasing filter (d) sampling with hamming window spatially constrained anti-aliasing filter (e) sampling with proposed slepian eigenfunction window spatially constrained anti-aliasing filter.

$\overline{w_{\ell}^{m}(k r)}=Y_{\ell}^{m}\left(\Omega_{t}\right) / b_{\ell}(k r)$ are the fourier coefficients of plane wave decomposition weights, $w$. Note that $b_{\ell}$ for the rigid sphere is calculated from [7]. The estimated pressure coefficients include aliasing and to remove the aliasing error, the coefficients are filtered by anti-aliasing filters described in section III-C using (11). The array directivity for various array look directions $\Omega_{t}$ is then calculated as

$$
y=\sum_{\ell=0}^{L-1} \sum_{m=-\ell}^{\ell}(\tilde{p})_{\ell}^{m}(k r) \overline{w_{\ell}^{m}(k r)},
$$

where $(\tilde{p})_{\ell}^{m}(k r)$ are the estimated pressure coefficients obtained after low pass effect of anti-aliasing filters.
In Fig. 3, we plot the array directivity for five different cases in form of polar plots. Fig. 3(a) shows array directivity when there is no aliasing error, that is, $(\tilde{p})_{\ell}^{m}=$ $(p)_{\ell}^{m}$. Directivity including aliasing error without using any anti-aliasing filter is shown in Fig. 3(b) where it is seen that there is a significant increase in the sidelobes due to the spatial aliasing introduced by significant high frequency harmonics. Fig. 3(c), Fig. 3(d) and Fig. 3(e) shows array directivity pattern when we perform spatial truncation of the ideal filter by using rectangular window, hamming window and eigenfunction window of width $\theta_{o}=44^{\circ}$ respectively. It is observed from the polar plots obtained by applying sptaially constrained anti-alisaing filters that there is a significant reduction in the sidelobes introduced by aliasing errors in Fig. 3(b). Also, spa- 
tially constrained anti-aliasing filter obtained by applying eigenfunction window provides higher attenuation and better results than the rectangular and hamming windows based spatially constrained anti-aliasing filters.

\section{B. White Noise Gain and Directivity Index}

In this section, we perform robustness analysis by measuring the white noise gain (WNG), which represents the improvement in signal to noise ratio at the array output. We have also measured the directivity index (DI) which gives a measure to improve directivity of the array compared to an omnidirectional microphone. Both the WNG and DI for $M \geq L^{2}$ microphones are calculated in the same way as in [12] and for 30 microphones, the values for different array outputs using different filters are shown in Table I. It can be seen that the proposed anti-aliasing filter spatially truncated by eigenfunction window of width $\theta_{o}$ exhibits superior properties than those truncated by rectangular and hamming windows of same width as WNG and DI values of the proposed eigenfunction filters are closer to the ideal filter.

TABLE I: Performance Parameters

\begin{tabular}{|c|c|c|}
\hline Filter & WNG & DI \\
\hline Ideal & 35.88 & 55.00 \\
Rectangular & 32.87 & 51.81 \\
Hamming & 34.48 & 53.78 \\
Slepian Eigenfunction & 35.39 & 54.22 \\
\hline
\end{tabular}

\section{Conclusions}

In this paper, we have designed a spatially constrained anti-aliasing filter by spatially truncating the ideal antialiasing filter applying the proposed Slepian eigenfunction window, in order to reduce the spatial aliasing error which affects the performance of the spherical microphone arrays at high frequencies. We have shown that aliasing error is reduced by the use of anti-aliasing filters. We compare the performance of the proposed filter with the spatially constrained filters obtained by spatially truncating ideal filter using rectangular window and hamming window of the same width. We have also shown that anti-aliasing filter obtained by applying the proposed eigenfunction window performs better in terms of WNG and DI than the one obtained after applying rectangular and hamming windows.

\section{REFERENCES}

[1] J. Meyer and G. Elko, "A highly scalable spherical microphone array based on an orthonormal decomposition of the soundfield," in 2002 IEEE International Conference on Acoustics, Speech, and Signal Processing, vol. 2, May 2002, pp. II-1781II-1784.

[2] D. L. Alon and B. Rafaely, "Beamforming with optimal aliasing cancellation in spherical microphone arrays," IEEE/ACM Transactions on Audio, Speech, and Language Processing, vol. 24, no. 1, pp. 196-210, Jan 2016.

[3] J. Meyer and T. Agnello, "Spherical microphone array for spatial sound recording," in Audio Engineering Society Convention 115. Audio Engineering Society, 2003.

[4] G. Weinreich and E. B. Arnold, "Method for measuring acoustic radiation fields," The Journal of the Acoustical Society of America, vol. 68, no. 2, pp. 404-411, 1980.

[5] T. D. Abhayapala and D. B. Ward, "Theory and design of high order sound field microphones using spherical microphone array," in 2002 IEEE International Conference on Acoustics, Speech, and Signal Processing, vol. 2, May 2002, pp. II-1949II-1952.

[6] R. A. Kennedy and P. Sadeghi, Hilbert Space Methods in Signal Processing. Cambridge, UK: Cambridge University Press, Mar. 2013.

[7] B. Rafaely, "Analysis and design of spherical microphone arrays," IEEE Transactions on speech and audio processing, vol. 13, no. 1, pp. 135-143, 2005.

[8] B. Rafaely, B. Weiss, and E. Bachmat, "Spatial aliasing in spherical microphone arrays," IEEE Transactions on Signal Processing, vol. 55, no. 3, pp. 1003-1010, 2007.

[9] Z. Khalid, R. A. Kennedy, and S. Durrani, "On the choice of window for spatial smoothing of spherical data," in Acoustics, Speech and Signal Processing (ICASSP), 2014 IEEE International Conference on. IEEE, 2014, pp. 2644-2648.

[10] Z. Khalid, S. Durrani, P. Sadeghi, and R. A. Kennedy, "Spatiospectral analysis on the sphere using spatially localized spherical harmonics transform," IEEE Trans. Signal Process., vol. 60, no. 3, pp. 1487-1492, Mar. 2012.

[11] F. J. Simons, F. A. Dahlen, and M. A. Wieczorek, "Spatiospectral concentration on a sphere," SIAM Rev., vol. 48, no. 3, pp. 504-536, 2006

[12] B. Rafaely, "Phase-mode versus delay-and-sum spherical microphone array processing," IEEE signal processing Letters, vol. 12, no. 10, pp. 713-716, 2005.

[13] Z. Li, R. Duraiswami, E. Grassi, and L. S. Davis, "Flexible layout and optimal cancellation of the orthonormality error for spherical microphone arrays," in 2004 IEEE International Conference on Acoustics, Speech, and Signal Processing, vol. 4, May 2004, pp. iv-41-iv-44 vol.4.

[14] J. D. McEwen and Y. Wiaux, "A novel sampling theorem on the sphere," IEEE Trans. Signal Process., vol. 59, no. 12, pp. 5876-5887, Dec. 2011.

[15] P. J. Kostelec and D. N. Rockmore, "FFTs on the rotation group," J. Fourier Anal. and Appl., vol. 14, pp. 145-179, 2008.

[16] F. J. Harris, "On the use of windows for harmonic analysis with the discrete fourier transform," Proceedings of the IEEE, vol. 66, no. 1, pp. 51-83, 1978. 